\title{
Magnetic resonance images related to clinical outcome after psychosurgical intervention in severe anxiety disorder
}

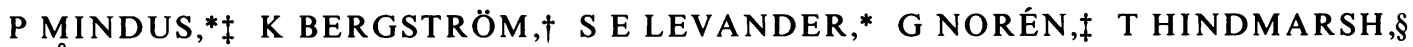 \\ K- $\AA$ THUOMAS $\dagger$ \\ From the Departments of Psychiatry, ${ }^{*}$ Neurosurgery $\ddagger$ and Neuroradiology,§ Karolinska Hospital, Stockholm, \\ and Department of Diagnostic Radiology, $\uparrow$ University Hospital, Uppsala, Sweden
}

SUMMARY Postoperative verification of radiosurgical lesions in white matter has been difficult to obtain with CT. With magnetic resonance (MR) imaging, however, lesions could be demonstrated in patients undergoing a psychosurgical procedure, gamma capsulotomy, for anxiety disorder. The appearance and location of the lesions were related to the irradiation dose and to the long-term clinical outcome studied prospectively by two independent evaluators who had not been involved in the selection or the treatment of the patients. Seven consecutive cases were examined. CT was also included for comparison reasons. Lesions were clearly visible with $M R$ in patients who improved after surgery. Conversely, lesions were inadequate in cases who did not benefit. MR proved to be more accurate than CT both in detecting the lesions and in defining their configuration. The obser vations argue for the use of MR for post-operative verification of radiosurgical lesions. MR may facilitate the determination of a clinically effective radiation threshold estimate for radiosurgica lesions, which should be of value for the planning of studies of this type of limbic system surgery

Magnetic resonance imaging (MRI) is a reliable diagnostic method in neurology, neurosurgery and psychiatry. MRI not only yields images comparable or superior to those of computed tomography (CT) but also offers the potential for a more accurate discrimination between healthy and diseased tissue, ${ }^{1}$ without exposing the patient to $x$-rays or to any other known health risk. ${ }^{2}$

Stereotactic radiosurgery is an effective neurosurgical method for non-invasive lesioning of cerebral pathways and relays, ${ }^{34}$ applied also to the field of psychosurgery. ${ }^{5}$ Gamma radiation from a stereotactic $60-$ Co unit is delivered to the white matter in the anterior limb of the internal capsule. The aim is to interrupt fronto-limbic pathways through the capsule $;^{5-7}$ hence the term of the intervention, gamma capsulotomy. However, a disadvantage has been that gamma lesions in white matter have not been reliably

Address for reprint requests: Dr P Mindus, Karolinska Hospital, S-104 01 Stockholm, Sweden.

Received 2 September 1986 and in revised form 16 February 1987. Accepted 24 February 1987 demonstrated with $\mathrm{CT}{ }^{5}$ Consequently, it has not been possible to establish with any degree of certainty? the relationship between the site and the size of the lesions and the therapeutic outcome of the intervention. In a recent pilot case, however, gamma lesions in the internal capsule could be demonstrated with MRI in the immediate postoperative period. ${ }^{4}$ We used MRI in an attempt to visualise lesions several years after gamma capsulotomy, and to relate their site and size both to the irradiation dose delivered, and to the long-term clinical outcome as determined prospectively by two independent evaluators. Moreover, CT was also performed to permit comparison between the two imaging methods.

Many earlier reports on psychosurgery have been difficult to evaluate owing to retrospective study designs, short duration of follow-up, subjective or unstandardised measures of outcome used, most commonly, by the physician responsible also for the selection and the treatment of the patients. This study was designed to avoid some of these short-comings. In this investigation seven consecutive patients were examined before and again 7 years after surgery by two psychiatrists who had not been involved in the selec- 
tion and the treatment of the patients. Several rating scales were used to monitor clinical outcome. Since psychosurgery is only carried out on a small number of patients we feel that a report on even this limited number of cases is justified.

\section{Patients}

Five female and three male consecutive patients were studied who had been operated upon with gamma capsulotomy during the years 1977 through 1979. The cases had been selected for the intervention by the late $G$ Rylander according to inclusion and exclusion criteria described elsewhere. ${ }^{9}$ The patients had been hospitalised repeatedly for their anxiety disorders, which had proved resistant to available conventional treatments. The median duration of their illness was 17 (range $=5-25$ ) years. All patients had been examined preoperatively by two of us (PM, SEL). The preoperative records were used, in retrospect, to diagnose the cases according to the criteria of the DSM III $^{8}$ which were not available at the initial interviews. The patients could be assigned to the following diagnostic groups: 300-21 Agoraphobia with Panic Attacks, 300.23 Social Phobia, 300.2 Generalised Anxiety Disorders and $300 \cdot 30$ ObsessiveCompulsive Disorder. Demographic and clinical data are shown in the table.

The patients median age at operation was 42 (range = 22-47) years. One male patient was excluded from the psychiatric follow-up examinations, since an additional capsulotomy with radiofrequency heat lesions had been performed 1.5 years after the gamma capsulotomy.

\section{Methods}

\section{Psychosurgical methods}

The patients were operated upon using the stereotactic gamma unit described by Leksell. ${ }^{34}$ Encephalograms and CT scans were used to localise the targets which were placed $10 \mathrm{~mm}$ in front of the anterior commissure, $8 \mathrm{~mm}$ above the inter-commissural line and on average $17 \mathrm{~mm}$ lateral to the mid-plane. ${ }^{5}$ This target was chosen according to specific considerations, ${ }^{67}$ and irradiated by the cross-firing of 179 precisely collimated, narrow $(3 \times 5 \mathrm{~mm})$ beams of $60-\mathrm{Co}$ gamma radiation. On the basis of experience gained from postmortem observations on patients subjected to gamma thalamotomy for cancer pain, ${ }^{10}$ an intended central irradiation dose of $160 \mathrm{~Gy}$ was chosen.

\section{Imaging methods}

MRI: The patients were examined with the 0.5 Tesla superconducting MR unit (Siemens Magnetom) at the University Hospital in Uppsala. The examination comprised the following spin echo sequences: $\mathrm{T} 1, \mathrm{~T} 2$, and proton density weighted sequences with TR and TE of 500/35 1500/120 and $1500 / 35 \mathrm{~ms}$ respectively in the axial and frontal planes (in one case the sagittal plane was also included). Multi-slice technique with a slice thickness of $5 \mathrm{~mm}$ was used. The two radiologists ( $K B$ and $K-\AA \AA T$ ) evaluating the $M R$ images were unaware of the individual patient's irradiation dose and clinical outcome. The conspicuity, the site and size of the lesions in the capsule were considered as well as any secondary changes around the lesions. A forced ranking of the MR images of each of the seven patients was made. A case demonstrating well circumscribed, bilateral lesions placed basally in the anterior limb of the internal capsule and displaying no significant other signs of brain pathology would be given rank 1 . A case with invisible or misplaced lesions or signs indicating significant brain pathology, for example, clear-cut atrophy, would be given rank 7 .

CT: To permit comparison between the MR and the CT imaging methods, the patients were examined also with high resolution CT scanners currently in use. No contrast medium was administered. Contiguous axial slices $1.5-5 \mathrm{~mm}$ thick, covering the internal capsule were obtained. The radiologist (TH) evaluating the CT images, and performing the comparison of the CT and MR images, was unaware of the results of the MRI ranking and of the psychiatric evaluation.

\section{Psychiatric evaluation}

Two psychiatrists (PM, SEL) examined the patients before and 6-8 (median $=7$ ) years after surgery. The evaluators had not been involved in the selection and the treatment of the patients. Both psychiatrists were unaware of the individual patient's irradiation dose and the neuroradiological findings until the study was completed. In addition to comprehensive interviews with the patients, a relative was also interviewed. It was made clear to all interviewed persons that it was important to report all changes including negative ones. Clinical morbidity was rated by both evaluators in simultaneous rating sessions using the Comprehensive Psychopathological Rating Scale (CPRS). This scale has been shown to be highly sensitive to symptom changes induced by psychiatric treatment. It has high validity, and high intraand inter-rater reliabilities. (For description of the scale and validity and reliability data, see ref 11 ).

The effect of the intervention on the patient's symptom

able Demographic, clinical, radiosurgical, and neuroradiological data on seven gamma capsulotomy cases

\begin{tabular}{|c|c|c|c|c|c|c|c|c|c|}
\hline \multirow[b]{2}{*}{ 'ase } & \multirow[b]{2}{*}{$\operatorname{Sex}$} & \multirow[b]{2}{*}{ Diagnosis } & \multirow{2}{*}{$\begin{array}{l}\text { Duration of } \\
\text { illness }(y r)\end{array}$} & \multirow{2}{*}{$\begin{array}{l}\text { Age at } \\
\text { operation } \\
(y r)\end{array}$} & \multirow{2}{*}{$\begin{array}{l}\text { Dose (Gy) } \\
\text { left/right }\end{array}$} & \multirow{2}{*}{$\begin{array}{l}M R I \\
\text { rank }\end{array}$} & \multirow{2}{*}{$\begin{array}{l}\text { Bilateral lesions } \\
\text { on } C T\end{array}$} & \multicolumn{2}{|c|}{ Score at follow-up } \\
\hline & & & & & & & & Pippard & Axis $V$ \\
\hline $\begin{array}{l}1 \\
- \\
1\end{array}$ & $\begin{array}{l}F \\
F \\
F \\
F \\
M \\
F \\
M\end{array}$ & $\begin{array}{l}300 \cdot 21 \\
300 \cdot 23 \\
300 \cdot 02 \\
300 \cdot 02 \\
300 \cdot 21 \\
300 \cdot 02 \\
300 \cdot 21\end{array}$ & $\begin{array}{r}25 \\
13 \\
16 \\
22 \\
5 \\
20 \\
12\end{array}$ & $\begin{array}{l}47 \\
37 \\
44 \\
47 \\
25 \\
41 \\
40\end{array}$ & $\begin{array}{l}160 / 160 \\
160 / 160 \\
160 / 160 \\
160 / 100 \\
160 / 160 \\
152 / 152 \\
120 / 120\end{array}$ & $\begin{array}{l}4 \\
1 \\
3 \\
7 \\
2 \\
6 \\
5\end{array}$ & $\begin{array}{l}\text { No } \\
\text { Yes } \\
\text { Yes } \\
\text { No } \\
\text { Yes } \\
\text { No } \\
\text { No }\end{array}$ & $\begin{array}{l}\mathbf{A} \\
\mathbf{A} \\
\mathbf{A} \\
\mathbf{C} \\
\mathbf{B} \\
\mathbf{C} \\
\mathbf{B}\end{array}$ & $\begin{array}{l}3 \\
3 \\
3 \\
6 \\
4 \\
6 \\
4\end{array}$ \\
\hline
\end{tabular}



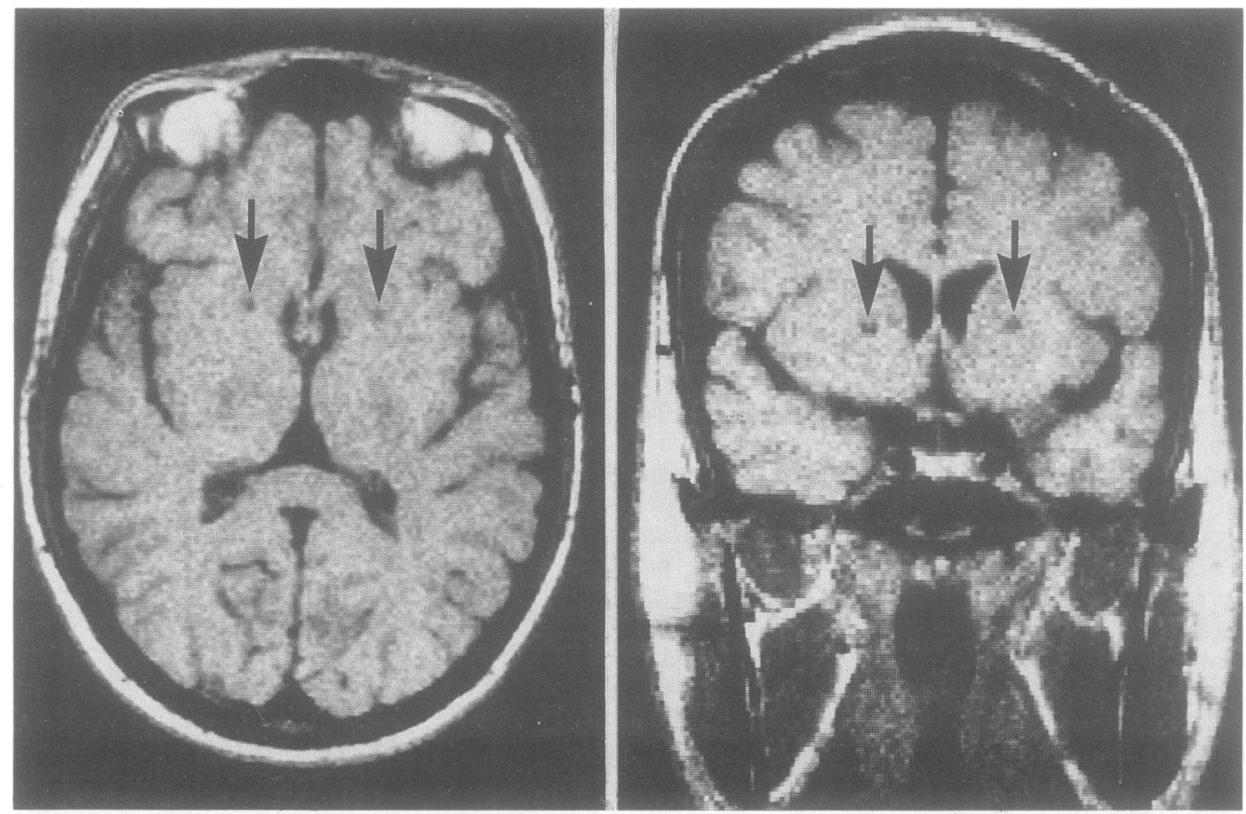

Fig 1 Case B. Radiosurgical lesions in the internal capsules (arrows) 7 years after psychosurgical intervention. $T_{1}$ weighted sequence, axial (left) and frontal planes.

was studied by comparing pre-operative and post-operative scores on the CPRS. Furthermore, the evaluators conjointly rated the patient on the Pippard post-operative rating scale widely used in the psychosurgery literature. With that scale the results are classified into five groups $A, B, C, D$, and E. ${ }^{12}$ According to international convention, outcome is judged as satisfactory in group A and B patients. ${ }^{7}$ In order to supplement the above symptom oriented outcome measures the patient's highest level of functioning in three areas (social relations, occupational functioning, and use of leisure time) during the year preceding the psychiatric follow-up examination was rated on the Axis V of the DSM III. ${ }^{8}$ The scale has the following seven steps: 1 Superior, 2 Very Good, 3 Good, 4 Fair, 5 Poor, 6 Very Poor, and 7 Grossly Impaired. (Details of the follow-up procedure will be published elsewhere.)

\section{Results}

The results are summarised in the table.

$M R I$ A radiosurgical lesion was demonstrated with MRI in all cases. They were most clearly visible in the Tl weighted images as areas with low signal intensity. In typical cases they appeared sharply defined (see fig 1). Their transverse and sagittal diameters averaged $6 \mathrm{~mm}$ and $13 \mathrm{~mm}$ in the axial projections. In five of the patients distinct bilateral lesions were found in the anterior limb of the internal capsule. In one case a cortical defect in the right occipital lobe was observed, indicative of a (clinically silent) infarction.
No other significant brain pathology was observed The tissue reactions in the vicinity of the lesions were optimally measured in the T2 weighted images, appearing as a diffusely delineated area with high sig nal intensity and thin bands radiating several milli meters from the lesions (fig 2 ). In the proton densityo weighted images neither the lesions nor the perifocaF changes were visualised as well as in the $\mathrm{T} 1$ and $\mathrm{T} 2$ weighted sequences. In one case (M), only a minimal lesion in the left internal capsule could be detected on the MR images. In another patient $(\mathrm{H})$ the lesion on the left side was smaller $(3 \times 3 \mathrm{~mm})$ than her right lesion which was of average size for the group. Therefore the MR images of patients $M$ and $H$ were given the ranks 7 and 6 .

$C T$ : Regions with low attenuation were observed bilaterally in the calculated target area in three cases and unilateral lesions were found in two cases. CT failed to demonstrate evidence of irradiation in cases $\mathbf{M}$ and $\mathbf{H}$. Reduction of the slice thickness generally improved visualisation of the lesions.

Comparison MRI-CT MRI demonstrated the size and site of the lesions better than did CT. Also, the accuracy in determining the configuration of the lesions was better for MRI than for CT. Moreover, lesions were visualised with MRI also in the instances in which CT was negative (see table).

Psychiatric results The interrater reliability for the CPRS ratings was highly significant (product moment 

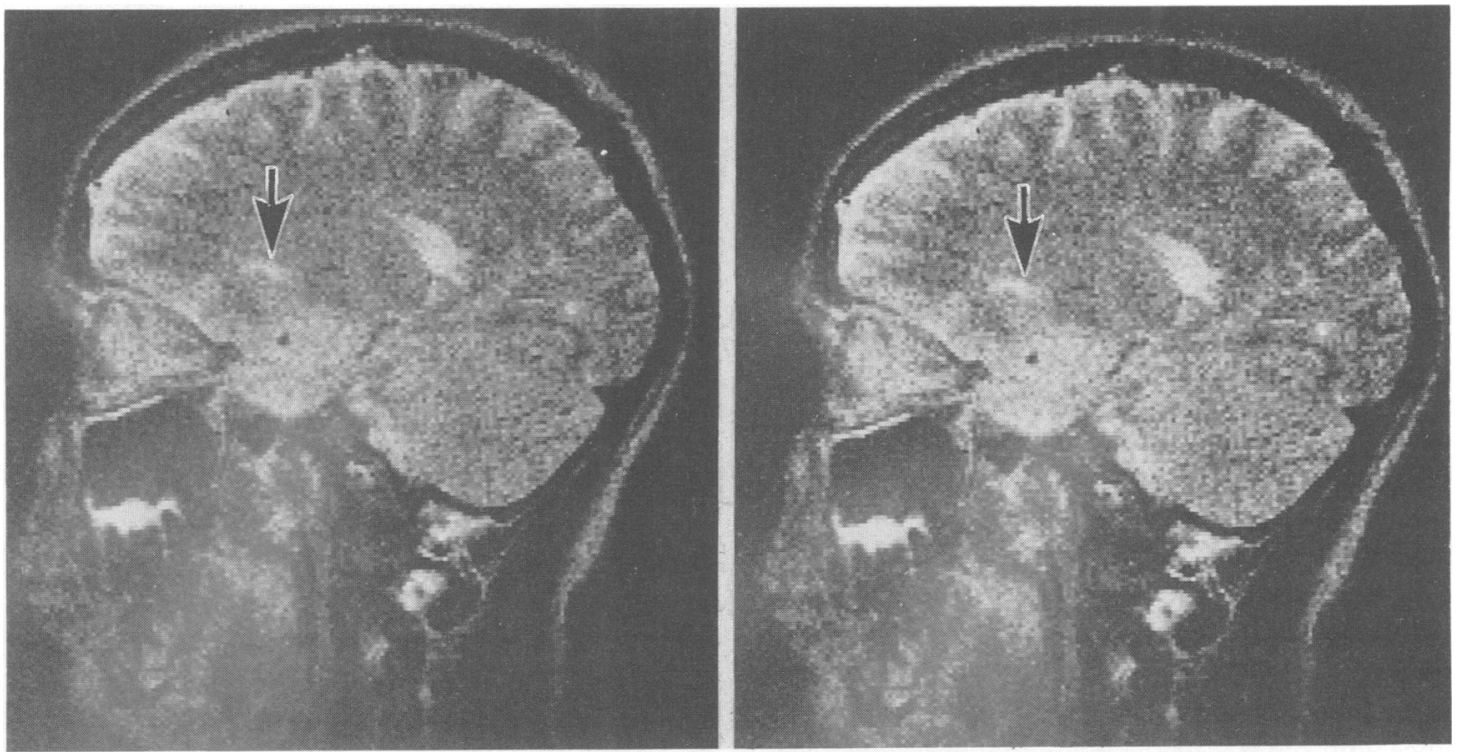

Fig 2 Case B. $T_{2}$ weighted image, sagittal planes covering the left and right targets.

correlation: 0.98). A one-way ANOVA of mean summed CPRS scores rated before and after surgery showed a highly significant reduction in symptom scores after surgery $(F(2.12)=14.9 p<0.001)$. The patient's score on the Pippard post-operative rating scale are shown in the table. Three patients were rated as $\mathrm{A}$, two as $\mathrm{B}$, two $(\mathrm{M}, \mathrm{H})$ as $\mathrm{C}$, and none as D or E. Thus, in five of the seven patients outcome may be judged as satisfactory. The patients' level of adaptive functioning during the year preceding the follow-up, that is, their Axis V score, is shown in the table. There was good agreement between the patients MRI rank, their scores on the Pippard scale, and their postoperative level of functioning.

Correlation between MRI and clinical outcome The MRI examination showed bilateral lesions in the internal capsule in all five patients who benefited from the operation. Conversely, MRI failed to visualise distinct, bilateral lesions in the two cases with unsatisfactory outcome. A strong correlation was found between the adequacy of the lesion and the postoperative level of social functioning (Kendall's tau $=0.81, \mathrm{p}<0.001$, see fig 3 ).

\section{Discussion}

The long-term therapeutic outcome of gamma capsulotomy, an anti-anxiety psychosurgical intervention, was determined prospectively by independent evaluators and related to the site and the size of the lesions demonstrated with MRI. In five of the seven cases $(70 \%)$, outcome was judged as satisfactory by the patients themselves, their relatives, and the evaluators. These clinical results agree well with those of previous reports on larger series. ${ }^{79}$ The present study is the first to show that radiosurgical lesions in white matter can be demonstrated with

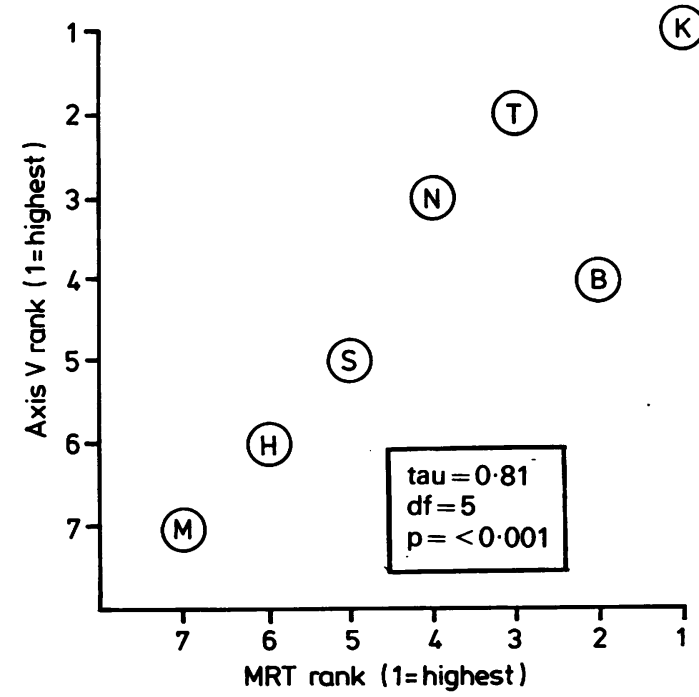

Fig 3 Post-operative social functioning in relation to accuracy of lesions. Axis $V$ rank was based on ratings of adaptive functioning. MRT rank was based on lesion characteristics demonstrated in the MR images. (tau = Kendall's tau for correlation between ranks.) 
MRI many years after irradiation. Despite the small number of cases studied a statistically highly significant correlation was obtained between clinical outcome and MRI findings.

The best pulse sequence for the demonstration of the radiosurgical lesion itself proved to be the one enhancing the $\mathrm{T} 1$ component of the images. The tissue reactions in the vicinity of the lesion, on the other hand, seemed to be more accurately assessed in the T2 weighted images. The tissue reactions accounting for the signal changes in the MR images are unknown, but it has been speculated that they represent a zone of "chronic oedema", perhaps induced by axonal degeneration. ${ }^{1314}$

The configuration of lesions produced with the same radiosurgical system and collimator apertures as those used in our study, but delivered to the grey matter of the thalamus in cancer pain patients, was examined in a post-mortem study. ${ }^{10}$ Lesions were found to be oval with the approximate diameters of $8 \mathrm{~mm}$ and $8 \mathrm{~mm}$ in the axial plane. This is in accordance with the size of the white matter lesions seen in the T1 weighted MR images in this study.

In 1979 Leksell and Backlund $^{5}$ failed to demonstrate lesions with CT performed 1-2 years after gamma capsulotomy. However, lesions were visible with CT in a majority of our patients, examined with modern high resolution CT scanners. The major reason for the discrepancy may be that the spatial and contrast resolutions of modern CT scanners are necessary to detect lesions of the actual size.

In comparing the two imaging methods it appears that MRI has a greater capability to demonstrate the gamma lesions, their configuration and the surrounding tissue reaction. Also, the facility to obtain MR images in many planes is another advantage. Furthermore, it may be assumed that radiosurgical lesions may be detected at an earlier stage after irradiation with MRI than with CT. This contention is supported by the demonstration of MRI signal changes 24 hours after gamma capsulotomy in the pilot case referred to above. ${ }^{4}$

After completion of the study the neurosurgical records were inspected. In case $\mathbf{M}$ it was revealed that a calculation error had been made during the right target localisation procedure. When this was observed by the neurosurgeon, the irradiation was interrupted when $100 \mathrm{~Gy}$ instead of the intended 160 Gy had been delivered. Inspection of patient H's record showed that the calculated dose of $152 \mathrm{~Gy}$ had been delivered. It has been shown that doses up to 160 Gy do not consistently cause radiosurgical lesions in grey matter. ${ }^{10}$ The minimal dose required to produce gamma lesions in white matter remains to be established. However, there is reason to believe that in cases $\mathbf{M}$ and $\mathbf{H}$ the dose delivered was not sufficient to produce lesions. This would explain not only their individual MRI findings, but also the clinical outcome, since it is well known that unilateral lesions are improductive. ${ }^{15}$

A collimator set with larger apertures had been selected for the last patient in the series (S). It may be calculated that with that set the cross section area of each beam would be more than doubled at the region of their intersection. The radiation dose was accordingly reduced to $120 \mathrm{~Gy}$ with the goal to obtain biological effects similar to those of the other patients, according to the "isoeffect concept". ${ }^{16}$ When judging from the MR images, this goal may have been attained, since the size and the configuration of the lesions in case $\mathrm{S}$ appeared similar to those of the other patients. The patient who was excluded from the psychiatric follow-up had been exposed to only 80 Gy. He did not benefit, and demanded an additional operation, this time with heat lesions. The second intervention was gratifying. It is of interest that MRI failed to demonstrate gamma lesions in his case.

In the gamma capsulotomy series reported by Rylander, ${ }^{9}$ two patients had been classified to group $\mathrm{C}$ and two to group D. Both D cases (now deceased) were exceptional in that they had undergone frontal lobe psychosurgery prior to gamma capsulotomy The two group $\mathrm{C}$ patients in his series were examined recently (by PM) and CT scans were made. In neitheo case could distinct, bilateral lesions be found. Thus in both series of patients inadequate lesions were noted in those cases who did not benefit from surgery.

Technical imperfections may be responsible for the treatment failures reported in this study, and pre sumably also for other psychosurgical failures reported elsewhere in the literature. The high correlations between clinical outcome and surgical accuracy, as reflected in the MR images, argues for the use of MRI to obtain post-operative verification. The study shows MRI to be more accurate than CT for that purpose.

It should be added that when determining the site of the lesions from the MR images, anatomical landmarks were used, not stereotactic coordinates. In the future, however, it should be possible to follow patients postoperatively with repeated MRI examination to study systematically the clinical effects of lesions placed stereotactically in various targets within the limbic system. Such studies should provide new information not only on the accuracy of the operative system in placing the lesion in the intended target, but also on a radiosurgical threshold estimate for clinically effective lesions, as well as the time course for the development of the lesions. This should be of value for the planning of interventions and follow-up studies in this type of limbic system surgery. 
The patients were operated on by doctors E-O Backlund, L Leksell, G Norén, and T Rähn.

This paper was supported in part, by the Swedish Medical Research Council (Projects No. 4545 and 6676) and presented, in part, to the XIII Symposium Neuroradiologicum, Stockholm, June 1986.

Dr Mindus is the recipient of the 1986 Upjohn Scholarship for psychiatric research and development awarded by the Swedish Psychiatric Association.

\section{References}

1 Brown RP, Kneeland B. Visual imaging in Psychiatry. Hosp Community Psychiatry 1985;36:489-96.

2 Bottomley PA. NMR in medicine. Comput Radiology 1984;8:57-77.

3 Leksell L. Stereotaxis and Radiosurgery: an Operative System. Charles C Thomas USA, 1971.

4 Leksell L, Herner T, Leksell D, Lindqvist C. Visualisation of stereotactic radiolesions by nuclear magnetic resonance. J Neurology, Neurosurg Psychiatry 1985;48:19-20.

5 Leksell L, Backlund E-O. Stereotactic gammacapsulotomy. In: Hitchcock ER, Ballantine Jr HR, Meyerson BA, eds. Modern Concepts in Psychiatric Surgery. Amsterdam: Elsevier/North-Holland Biomedical Press 1979.

6 Nauta WJH. Connections of the frontal lobe with the limbic system. In: Laitinen LV, Livingston KE, eds. Surgical Approaches to Psychiatry. Lancaster: MTP Publications. 1973:303-4.

7 Bingley T, Leksell L, Meyerson BA, Rylander G. Longterm results of stereotactic anterior capsulotomy, in obsessive-compulsive neurosis. In: Sweet WH, et al, eds. Neurosurgical Treatment in Psychiatry, Pain and Epilepsy. Baltimore: University Park Press, 1977.

8 DSM III. Diagnostic and statistical manual of mental disorders. 3rd ed. Washington: American Psychiatric Association 1980.

9 Rylander G. Stereotactic radiosurgery in anxiety and obsessive-compulsive states: Psychiatric aspects. In: Hitchcock ER, Ballantine Jr HR, Meyerson BA, eds. Modern Concepts in Psychiatric Surgery. Amsterdam: Elsevier/North-Holland Biomedical Press 1979: 235-40.

10 Steiner L, Forster D, Leksell L, Meyerson BA, Boetius J. Gammathalamotomy in intractable pain. Acta Neurochirurg 1980;52:173-84.

11 Asberg M, Montgomery S, Perris C, Schalling D, Sedvall G. CPRS-The Comprehensive Psychopathological Rating Scale. Acta Psychiatr Scand (suppl) 1978;271: 5-27.

12 Pippard J. Rostral leucotomy: a report on 240 cases personally followed up after $1 \frac{1}{2}$ to 5 years. J Ment $S c i$ 1955;101:756-73.

13 Sheline GE, Wara WM, Smith V. Therapeutic irradiation and brain injury. Int J Radiat Oncol Biol Phys 1980;6:1215-28.

14 Caveness WF. Pathology of radiation damage to the normal brain of the monkey. Natl Cancer Inst Mongr 1977;46:57-76.

15 Meyer A, Beck E. Prefrontal Leucotomy and Related Operations-Anatomical Aspects of Success and Failure. London: Oliver and Boyd, 1954.

$16 \mathrm{Kjellberg} \mathrm{RN}$. Isoeffective dose parameters for brain necrosis in relation to proton radiosurgical dosimetry. In: Szikla G, ed. Stereotactic Cerebral Irradiation. INSERM Symposium no 12. Amsterdam: Elsevier/ North Holland Biomedical Press 1979. 